\title{
Low-temperature dynamics in a dye-doped polymer: correspondence between the data obtained by photon echo and single molecule spectroscopy
}

\author{
Kamil Karimullin ${ }^{1,2, *}$ and Andrei Naumov ${ }^{1,2}$ \\ ${ }^{1}$ Institute for Spectroscopy, 108840 Troitsk, Moscow, Russia. \\ ${ }^{2}$ Moscow State Pedagogical University, 119435 Moscow, Russia
}

\begin{abstract}
Low temperature dynamics (tunneling and vibrational relaxation) in doped polyisobutylene film has been reinvestigated using 2pulse incoherent photon echo (2IPE) and compared with single-molecule spectroscopy (SMS) data. It has been shown that in a very wide range of low temperatures the 2IPE gives optical dephasing times which correspond to the narrowest zero-phonon lines of single dye molecules.
\end{abstract}

Combined photon echo (PE) and single-molecule spectroscopy (SMS) technique is a very powerful tool for detailed study of low-temperature dynamics in dye-doped disordered polymers and glasses [1]. However this type of studies reveal discrepancies when comparing dephasing times $\mathrm{T}_{2}$ and single-molecule (SM) zero-phonon spectral lines (ZPL) width obtained from PE and SMS, correspondingly. For tetra-tert-butylterrylene (TBT) in polyisobutylene (PIB) PE-experiments obtained $\mathrm{T}_{2}$-times much shorter than it is sufficient to cause the corresponding ZPL broadening in the temperature range of a few - dozen of Kelvins [2, 3]. We experimentally solved this contradiction in Ref. [4]. In this paper we show that in temperature range from 4.5 up to $20 \mathrm{~K}$ the 2-pulse incoherent PE (2IPE) technique gives $\mathrm{T}_{2}$-times which correspond the narrowest SM ZPL.

The new series of PE-measurements were performed using the home-build 2IPE spectrometer that was constructed originally in the mid of 1990ies [1, 5], and recently reconstructed to achieve modern experimental possibilities $[4,6]$. We used a thin $(0.5 \mathrm{~mm})$ film of PIB with TBT molecules. PIB $\left(\mathrm{M}_{\mathrm{w}}=4.2 \times 10^{6} \mathrm{~g} \cdot \mathrm{mol}^{-1}\right.$, Sigma Aldrich) was dissolved in spectrally pure toluene (Sigma Aldrich) together with TBT. The sample was prepared by disposing of the solution between two glass slides and further pumping for toluene removal.

We measured 2-pulse IPE (2IPE) decay curves at different cryogenic temperature, and obtained the temperature dependence of the inverse optical dephasing time $1 / \pi \mathrm{T}_{2}$. This dependence is shown in Fig. 1 (large solid circles) together with previous 2IPE-data as measured originally in [7] (solid squares); T-dependences of ZPL for several single TBT molecules in PIB (small colour dots), as measured in [8]; and T-dependence of average spectral ZPL widths for a number of TBT SM in PIB (blue stars) as measured in [9].

In both experiments $[8,9]$ the fluorescence excitation spectra of single TBT molecules in thin $(0.5-1 \mu \mathrm{m})$ PIB film were measured by tuning of frequency of narrowband (1 -

* Corresponding author: kamil@isan.troitsk.ru 
$2 \mathrm{MHz}$ ) dye CW-laser and detection of Stokes-shifted luminescence. In Ref. [8] the confocal scheme together with avalanche photodiode (time of measurement for one spectrum was $t_{\mathrm{m}}=250 \mathrm{~s}$ ), whereas in Ref. [9] the wide-field luminescence microscope was used with detection by EM CCD camera $\left(t_{\mathrm{m}} \sim 10 \mathrm{~s}\right)$.

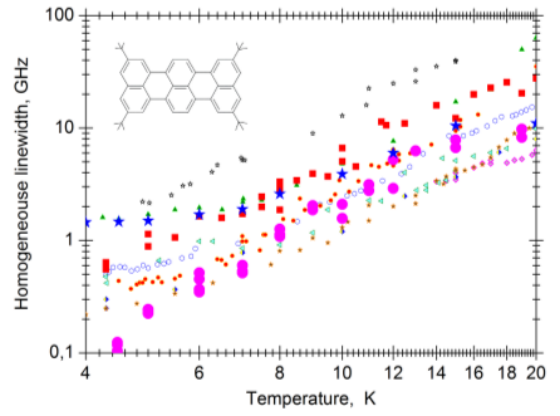

Fig. 1. Temperature dependences of $\mathrm{ZPL}$ homogeneous width measured by means of 2IPE (solid circles - new data, solid squares - previous data) and SMS (small dots and stars).

While the effective $t_{\mathrm{m}}$ for 2IPE $(\sim 10 \mathrm{~ns})$ is much less than the effective $t_{\mathrm{m}}$ in SMSmeasurements $(\sim 250 \mathrm{~s})$, the average value of $1 / \pi \mathrm{T}_{2}$ should be in the region of narrowest $\mathrm{SM}$ ZPL widths. It is exactly what was observed in the comparative SMS/PE-studies at $\mathrm{T}=2 \mathrm{~K}$ [8]. At higher temperatures $(4-20 \mathrm{~K})$ the ZPL broadening (as well as optical dephasing) is mainly caused by electron-phonon coupling, although the spectral diffusion processes are also intensified with $T$. Again, the value of $1 / \pi \mathrm{T}_{2}$ should be in the region of the narrowest ZPL widths. However, this was not observed in previous studies $[1,7,8]$ for a broad range of temperatures. If we compare previous $\mathrm{PE}$ data with SM and average SM ZPL widths, we see that $1 / \pi T_{2}$ comparable or even larger than average SM ZPL widths, that has not reasonable explanation.

We have carefully re-measured dephasing times for TBT in PIB in a very wide range of cryogenic temperatures from $4.5 \mathrm{~K}$ up to $20 \mathrm{~K}$. The high sensitivity of the updated 2IPEsetup allows one to eliminate any saturation and light induced effects. As one can see from the Fig. 1 the new 2IPE data are in reasonable agreement with theoretical considerations. The $1 / \pi \mathrm{T}_{2}$ values correspond to the narrow ZPLs.

As possible explanations for the question why the previous PE-data did not agree with SMS, we can suggest the following two: (1) The additional contributions into the effective dephasing processes were observed to be due to high laser excitation power. Now the modern detectors and adjustment optical scheme [6] gives opportunity to diminish the laser pulse energy enough to obtain unsaturated values of $1 / \pi T_{2}$. (2) Second possible reason is difference of molecular weight of PIB used in PE- and SMS-data. As it is known now [10] when diminish the $\mathrm{M}_{\mathrm{w}}$, additional spectral diffusion processes are strongly activated. As information about molecular weight of PIB is absent in first original PE researches, one can assume, that it was PIB with lower $\mathrm{M}_{\mathrm{w}}$ in comparison with SMS-experiments, that leads to larger values of $1 / \pi T_{2}$ measured by IPE. In this case the main role plays the spectral diffusion processes in nanosecond time scale.

The work was supported by the RFBR (project \# 18-02-01121).

\section{References}

1. K.R. Karimullin, A.V. Naumov, J. Luminescence, 152, 15 (2014)

2. A.V. Naumov et al., Phys. Stat. Sol. B, 241, 3480 (2004)

3. A.V. Naumov et al., Phys. Stat. Sol. B, 241, 3487 (2004)

4. M.V. Knyazev, K. Karimullin, A. Naumov, Phys. Stat. Sol. RRL, 11, 1600414 (2017)

5. Y.G. Vainer et al., J. Luminescence, 86, 265 (2000)

6. K.R. Karimullin et al., Meas. Science and Technology, 24, 027002 (2013)

7. S.J. Zilker et al., J. Luminescence, 76-77, 157 (1998)

8. Y.G. Vainer, A.V. Naumov, M. Bauer, L. Kador, J. Chem. Phys., 122, 244705 (2005)

9. A.A. Gorshelev et al., Chem. Phys. Chem., 11, 182 (2010)

10. I.Y. Eremchev et al., Phys. Chem. Chem. Phys., 13, 1843 (2011) 